\title{
Particulate lubricants in cosmetic applications
}

K Timm ${ }^{1,3}$, C Myant $^{2}$, H A Spikes ${ }^{2}$, M Grunze $^{3}$

1. Beiersdorf AG, Research \& Development, Unnastraße 48, 20245 Hamburg, Germany

2. Tribology Group, Department of Mechanical Engineering, Imperial College London, SW7 2AZ, UK

3. Angewandte Physikalische Chemie, Universität Heidelberg, Im Neuenheimer Feld 253, Heidelberg, Germany 


\begin{abstract}
Polymer powders are commonly added to cosmetic formulations to improve product performance and skin feel. This study investigates the effect of particle concentration and size on the lubricating properties of powder suspensions. Results are reported for various particle sizes and concentrations.

When the tribological contact was fully immersed the addition of particles had no effect. However different behaviour was observed when the contact was only partially-lubricated. In this case, a three stage friction coefficient curve was observed. By varying the particle size and concentration it was shown that the duration and magnitude of each stage can be controlled.
\end{abstract}

Key Words: sliding friction; elastomer; particulate; real contact area 


\section{Introduction}

Particulate lubricants, such as mineral oils containing dispersed solids, milling pastes and machining lubes, are extensively used in industrial applications. It has been shown that such solids may be beneficial or deleterious in terms of lubricant performance, depending upon the nature of the particles and on the operating conditions employed. ${ }^{[1]}$ Particles larger than the minimum film thickness of a lubricated contact can cause unwanted damage to surfaces and lead to early mechanical failure of machinery elements, even if the particles are softer than the rubbing surfaces. ${ }^{[1]}$ Suspended solids having a layer-lattice structure such as graphite and $\mathrm{MoS}_{2}$ have been shown to reduce friction in steel-steel contacts. ${ }^{[2]}$ Because of its focus on industrial application, the majority of lubricant particulate research has focused on the lubrication of hard contacts, such as occurs between most metal surfaces.

Little is known about the behaviour of particulate lubricants in a compliant contact where one or both of the contacting surfaces are of lower elastic modulus than the particles. Examples of the latter include the mastication of food, eye irritation caused by dirt, the presence of stones in a road-tyre contact, and the use of particles in cosmetic products applied on the skin.

It is common for cosmetic companies to use descriptive panels with trained panellists as well as consumer studies to evaluate their products' performance. But these tests are costly, timeconsuming and subjective. ${ }^{[3]}$ There is currently considerable interest to develop ways to improve the measurement of tactile perception. ${ }^{[4]}$ The aim is to objectify the assessment by measurement of physical parameters that determine the skin feel of products. ${ }^{[3]}$ Only selected products would then have to be submitted to extensive field tests. Moreover, such measurements would inform the design of attractive, desirable products. ${ }^{[4]}$ A wide variety of cosmetic powders are commercially-available, which differ in shape, size, compressibility, and oil absorption. Understanding how these properties affect the powder's performance on skin would provide guidance to developers of cosmetic and pharmaceutical formulations. ${ }^{[5]}$

It has been shown that the tribological properties of cosmetic products affect the latters' performance ${ }^{[6,7]}$ and skin feel. ${ }^{[5,8,9]}$ To date, friction tests on lubricated skin have focussed on the effects of skin hydration ${ }^{[9-13]}$, commercial skin creams ${ }^{[5,6,9]}$, and cosmetic ingredients such as petrolatum and glycerol. ${ }^{[6,9]}$ It has also been shown that, in dry contacts, talcum powder reduces friction in compliant contacts, ${ }^{[14]}$ and on skin. ${ }^{[12,13,15]}$ Gerrard found that the reduction of friction by application of talcum powder was only significant under sufficiently 
humid conditions. ${ }^{[16]}$ In line with this finding, Comaish observed that application of talcum powder on hydrated skin resulted in higher friction values than talcum powder on dry skin. ${ }^{[13]}$ However, the exact amounts of water and talcum applied to the skin were not specified in the vast majority of these studies.

To the authors' knowledge, the lubricating properties of cosmetic powders in suspensions have not yet been investigated and there has been no systematic approach to examine the influence of powder concentration and size. In the current study, friction was measured after application of suspensions of well-defined, spherical polymer powders; the effects of particle concentration and size were investigated. Particles were added to a simple, aqueous suspension medium rather that a commercially available cream so as to focus on the specific effect of particle addition.

An in vitro system was employed based on a ball-on-disc tribometer with a compliant contact formed between a polydimethylsiloxane (PDMS) ball and PDMS disc. PDMS is widely used to mimic bio-surfaces, as its mechanical properties are similar to those of human tissue ${ }^{[17]}$ and skin. ${ }^{[18]}$ Results are presented for the tribological contact of interest under dry and lubricated conditions. The influence of the aqueous suspension medium and a powder suspension on friction is investigated and lubrication mechanisms are discussed. Results are reported for various particle concentrations and sizes.

\section{Materials and Methods}

\subsection{Experimental procedure}

Friction measurements were carried out using a mini-traction machine (MTM; PCS instruments, London, UK), shown schematically in Fig. 1. The MTM provides fullyautomated friction mapping of fluids under hydrodynamic, mixed and boundary lubrication. Normally, the MTM uses metal balls and discs, but for the current study, a compliant contact was obtained by loading a PDMS or Nylon ball, $19 \mathrm{~mm}$ in diameter, against a moulded PDMS disc. The discs were $46 \mathrm{~mm}$ in diameter and $4.5 \mathrm{~mm}$ thick and were clamped on the top of a supporting, stainless steel disc.

The test protocol was as follows. For each test, the MTM ball and disc were cleaned with deionised water and isopropanol and left to air dry. For partially-lubricated (PL) conditions, $25 \mu \mathrm{l}$ of test lubricant were applied drop-wise using an Eppendorf pipette (Eppendorf AG, 
Hamburg, Germany) and immediately spread via the operator's finger covered with a sterile finger glove (AMPri GmbH, Stelle, Germany), resembling a cosmetic application. For fullyflooded (FF) contacts, the test fluid reservoir surrounding the disc was filled up with ca $30 \mathrm{ml}$ of lubricant, ensuring that the disc was fully covered with lubricant throughout the test. Measurements were carried out in an air conditioned room to ensure a relatively constant temperature $\left(18 \pm 2{ }^{\circ} \mathrm{C}\right)$ and humidity $(43 \pm 3 \%)$. The absolute value of friction coefficient was observed to be highly sensitive to temperature, humidity and the presence of air drafts; however the behavioural trends presented in this paper were found to occur under all experimental conditions.

Tests were carried out under conditions similar to those experienced during descriptive panel testing. An applied load of $0.5 \mathrm{~N}$ was employed, as this is suggested to be the standard exploratory load. ${ }^{[18]}$ Tests were performed under pure sliding conditions, by keeping the ball stationary and rotating the disc at a sliding speed of $50 \mathrm{~mm} / \mathrm{s}$. All tests were conducted for 20 minutes and repeated at least twice; average values are depicted in the figures showing friction coefficient versus time.

Since it was impossible to visualise the contact interface during tribometer experiments, tests were stopped at desired time points and the PDMS samples removed. Images of the contact area on the ball and disc were then taken using a colour CCD-camera and microscope setup with a $5 \mathrm{X}$ magnification lens. Due to the large contact size, multiple images of the contact were captured and then stitched. This was carried out in order to gain insights about the distribution of particles at different stages during the sliding test.

\subsection{Contacting surfaces}

The tribological contact was formed between a ball and a disc made of PDMS or Nylon. PDMS samples were fabricated from a component silicone kit (Sylgard 184, Dow Corning Corporation, USA). Sylgard 184 is supplied as a two part silicone elastomer kit. A base and a curing agent with mass ratio of 10:1 were used for all PDMS samples. The Young's modulus and Poisson's ratio of unmodified Sylgard 184 has been reported to be $2.2 \mathrm{MPa}^{[18]}$ and $0.49^{[19]}$ respectively which lies within the range of reported elasticity for human skin of 1-4 MPa. ${ }^{[20]}$ Using a Dynamic Mechanical Analysis (DMA) technique ${ }^{[21]}$ the Young's modulus of all PDMS samples used was found to be $2.7 \mathrm{MPa}$ with a loss tangent, $\tan \delta$, of 0.11 for the test conditions employed. The specific surface energy of adhesion, $\Delta \gamma$, between glass and PDMS 
was found to be $0.0356 \mathrm{~J} / \mathrm{m}^{2}$. ${ }^{[22]}$ The surfaces of skin and PDMS are both hydrophobic. Water droplet contact angles were measured (OCA 20, DataPhysics Instruments GmbH, Filderstadt, Germany) and found to be $95^{\circ}$ and $110^{\circ}$ for skin and PDMS, respectively. An MTM Nylon ball (PCS-Instruments) was used in one test and representative values of Young's modulus of 2.65 GPa and Poisson's ratio 0.39 were employed for calculations using this material. ${ }^{\text {[23] }}$

Surface roughness values for balls and discs were obtained using an optical profilometer (Veeco Instruments Inc, Plainview, New York, USA). Measurements were taken in the VSI mode and the camera was setup with an X20 objective lens. Images were 640 x 450 pixels, which equates to a field of view of ca $575 \times 430 \mu \mathrm{m}$. No filtering was used during measurements. An rms value of $501 \pm 20$ and $24 \pm 5 \mathrm{~nm}$ was found for the PDMS ball and the disc, respectively. An rms value of $650 \pm 50 \mathrm{~nm}$ was found for the Nylon ball.

\subsection{Cosmetic powders}

Nylon-12 particles (Arkema, Colombes, France) having three different size ranges were used as supplied. Particle size distributions were determined using a Mastersizer 2000 (Malvern Instruments Ltd., Malvern, UK), at least twice per sample. Polydispersity is expressed by the span according to British Standards [\#BS 2955]. The particle size distributions of the Nylon powders used in this study were found to be $11.5 \mu \mathrm{m}+/-0.6 \mu \mathrm{m}, 21.8 \mu \mathrm{m}+/-0.6 \mu \mathrm{m}$, and $63.2 \mu \mathrm{m}+/-0.6 \mu \mathrm{m}$ (mean diameter $+/$ - span). The particles clearly differ in size and the small span indicates a narrow particle size distribution.

Morphological analysis of Nylon-12 particles was performed by scanning electron microscopy (SEM) at $10 \mathrm{kV}$ using a LEO 1530 Gemini (Carl Zeiss SMT AG, Oberkochen, Germany). All sized particles had similar morphology, as illustrated in Fig. 2.

\subsection{Test lubricants}

All powders were suspended in the same aqueous suspension medium (SM) using a laboratory blender (Waring Products, Inc., Torrington, Connecticut, USA) at full speed for 30 seconds at room temperature. To prepare the SM, two master mixes were used. For the first, preservative agents, phenoxyethanol and methylparabene, were added to fully desalted water in a beaker and stirred. Then polyacrylic acid rheology modifier (Carbopol 980) was added and the mixture was left overnight to swell. Then sodium hydroxide was used for neutralization. Carbopol-neutralized physical gels are used as rheology modifiers (thickening agents) in numerous applications, since they are inexpensive, transparent, harmless, and easy 
to prepare and to clean. In parallel, a second master mix was made by adding Polysorbate 20 emulsifier to fully desalted water and stirred the blend for approximately one hour.

Equal proportions of the two above master mixes were then blended and stirred with a magnetic stirrer for an hour at room temperature to produce the SM. Therefore, in addition to water and particles, each final test sample contained rheology modifier $(0.1 \%$ wt.), emulsifier $1 \%$ wt.) and preservative. The powder content varied between 0 to $30 \%$ wt.

Rheology of the samples was determined using a parallel plate AR-G2 rheometer (TA Instruments, Philadelphia, USA) under ambient conditions $\left(25^{\circ} \mathrm{C}\right)$. Across the shear rate range tested $\left(10^{-1}\right.$ to $\left.10^{4} \mathrm{~s}^{-1}\right)$, the $\mathrm{SM}$ and all powder suspensions were found to be shearthinning. This behaviour is well-known for liquids containing Carbopol rheology modifier ${ }^{[24}$ ${ }^{25]}$ and for suspensions in general. ${ }^{[26,27]}$ All the samples had high shear rate viscosity in the range $\mathrm{ca} 0.01$ to $0.06 \mathrm{~Pa}$ s. The lubricant film thickness and, therefore shear rate, for the tribological contact of interest is unknown. It is likely that the true shear is close to or greater than $10^{4} \mathrm{~s}^{-1}$. As a result, for all numerical calculations it is assumed that all samples have a viscosity of $0.06 \mathrm{~Pa}$ s.

\section{Results}

\subsection{Friction tests in the absence of particles}

Tests without particles were conducted first, to obtain reference plots. These enabled the reader to identify clearly the effect of the particles' presence on friction.

Friction coefficient over time was measured initially under dry conditions, (with no lubricant added), and then lubricated by SM, (i.e. in the absence of particles) in both under fully flooded (FF) and partially-lubricated conditions. In fully-flooded conditions the contact was immersed by the SM throughout the measurement while under partially lubricated (PL) conditions, $25 \mu \mathrm{l}$ of the SM was spread around the disc track prior to testing. The latter condition is believed to resemble the process of applying a cosmetic product on skin. The friction results are shown in Fig. 3.

For the un-lubricated contact, a high, constant friction coefficient of $c a 0.44$ is observed. When lubricated with the SM under FF conditions, a clear reduction in friction coefficient to a value of $c a 0.012$ is observed. It is estimated that under these conditions the contact is operating in the isoviscous-EHL regime. Under PL conditions a three stage curve can be seen 
where friction coefficient values are first similar to FF values (stage 1). After ca 200 seconds a transition in friction coefficient occurs (stage 2), as friction rises from the FF value towards the value of the un-lubricated contact. A rapid increase is observed for the initial $c a 20$ seconds of this transition. As friction coefficient approaches values similar to the unlubricated case, the rate of increase slows until a final, relatively constant, upper friction coefficient plateau is reached (stage 3), which is similar to the value measured for the unlubricated contact.

\subsection{Effect of suspended particles on friction}

To investigate the effect of cosmetic particles, the friction coefficient was measured for the contact of interest, lubricated with a powder suspension. Nylon particles of $21.8 \mu \mathrm{m}$ diameter at $30 \%$ wt were used for the initial test suspension. The contact was tested under PL and FF conditions. The results are presented in Fig. 4, together with the particle-free friction curves from Fig. 3.

It is seen in Fig. 4 that a very slight reduction in friction coefficient occurs on addition of particles to the SM, under FF conditions. This reduction was almost independent of particle size and concentration (data not shown). In the PL test, the presence of the particles clearly has a large effect on the friction coefficient. The magnitude and period of each stage of the friction curve is altered as follows.

- Stage 1 , where $\mu$ is close to FF values, is lost entirely.

- Stage 2, the transition, now starts immediately and lasts much longer

- Stage 3 appears to be reached after approximately the same time as in the particle-free case but the final upper value of the friction coefficient is reduced.

A series of tests of differing duration were carried out and images were taken of the contact region on the ball and of the disc track at the end of each test. Figure 5 shows the contact region on ball and disc at various stages during PL suspension tests matching positions (a) to (e) in Fig. 4.

At the beginning of the test, $t=0 \mathrm{~s}$ (a), it can be seen that a large number of particles are present within the middle of the disc track and on the contact region on the ball. After $\mathrm{ca} 20$ seconds (b) a large proportion of the particles present on the disc track have already been pushed to the track edges. At this point, the onset of friction increase from FF to dry state has begun. As friction rises further at (c), the majority of the particles have been removed 
completely from the contact area on both ball and disc. On the ball, the particles are concentrated at the contact inlet, suggesting that few particles are passing through the contact. There is little change to the particle distribution from this point onwards; however friction continues to rise for a further $c a 250 \mathrm{~s}$ (d). Images captured at the end of the test (e) show that the majority of the particles have been removed from the contact area.

The particles appear to concentrate around the contact region on the ball, i.e. the stationary surface, while relatively few of them can be seen at the edges of the track on the moving disc. This phenomenon has been observed by other investigators ${ }^{[28]}$ studying industrial processes involving the passage of material through the nip between rollers; such as paper manufacture, rubber processing and metal rolling. The transfer was found to depend on the ratio of surface speeds and the amount of compression through the nip. For small compressions the material always adhered to the slower surface, but for large compressions the opposite was true. As the particles exited the nip they separated from the interface at which the adhesive strength was least; where the intensity of shear stress was greatest. For the pure sliding contact of interest this will always be the interface with the moving surface.

\subsection{Effect of particle concentration and size}

To determine the effect of particle concentration on the lubricating properties of the suspensions, six different concentrations of $21.8 \mu \mathrm{m}$ Nylon particles were tested. Figure 6 shows the effect of particle concentration on the friction coefficient over the test period together with the particle-free friction curves from Fig. 3.

It can be seen that particle concentration has negligible effect on the initial friction coefficient value and the duration of stage 3 . However, with increasing particle concentration;

- the duration of stage 1 is decreased

- the duration of stage 2 is increased

- slope of the fricton curve in the transition region increases

- A small decrease in the final friction coefficient is observed.

For particle concentrations greater than $25 \% \mathrm{wt}$, no stage 1 behaviour is observed.

Figure 7 presents images of the ball taken after the test on suspensions containing three different powder concentrations. An increase in lubricant particle concentration corresponds 
to a larger accumulation of powder around the contact and in the inlet region. Pictures of the corresponding area on the disc do not show any significant differences in particle distribution.

Figure 8 shows the effect of particle size on the friction coefficient over the test period. All suspensions contained $30 \% \mathrm{wt}$ of powder, while mean particle diameters were 11.5, 21.8 and $63.2 \mu \mathrm{m}$. For all three suspensions, the friction coefficient appears to increase immediately after the start of the test, i.e. there is no stage 1 behaviour. A clear relationship between the rate of transition and particle size is observed. Smaller particle diameter results in both a longer transitional period and lower final friction coefficient.

Also presented in Fig. 8 is the friction trace from an un-lubricated test where a PDMS disc was rubbed against a Nylon ball, used instead of a PDMS ball. This was conducted to identify any differences in friction between PDMS on PDMS and Nylon on PDMS. The result shows that friction coefficient is $c a 6$ times lower for a Nylon/PDMS contact compared to a PDMS/PDMS contact. This suggests that, in principle, if Nylon particles are introduced into a PDMS/PDMS contact and support load, friction should be reduced from a high PDMS/PDMS value towards the lower Nylon/PDMS friction value.

Figure 9 shows photomicrographs of the contact region on ball and disc, captured at the end of PL tests with suspensions of Nylon particles of different sizes, i.e. 11.5, 21.8, and $63.2 \mu \mathrm{m}$, all at $30 \%$ wt. It can be seen in Fig. 9 that only very few particles of $63.2 \mu \mathrm{m}$ diameter are left in the contact region. By contrast, a large number of particles of $11.5 \mu \mathrm{m}$ diameter are still visible. The larger particles appear to accumulate further away from the middle of the track. Figure 9 also shows that particles of $63.2 \mu \mathrm{m}$ diameter accumulate at the contact outlet whereas the majority of the smaller particles accumulate at the inlet. The reason for this is not clear.

\section{Discussion}

\subsection{Friction between dry surfaces}

It is generally accepted that the friction of un-lubricated surfaces originates from two main sources. ${ }^{[29]}$ According to the two-term, non-interaction model developed by Bowden and Tabor, ${ }^{[10,30]}$ the total friction coefficient, $\mu_{t o t}$, can be described as the sum of an interfacial adhesion term, $\mu_{\text {int }}$, and a deformation term, $\mu_{\text {def }}$;

$$
\mu_{\text {tot }}=\mu_{\text {def }}+\mu_{\mathrm{int}}
$$


The interfacial adhesion term corresponds to the energy dissipated in the rupture of intermittent junctions formed between the two sliding surfaces due to short-range molecular attractive forces, such as van der Waals interactions. This equates to the real area of contact, $A$, multiplied by the interfacial shear stress, $S\left(\mu_{i n t}=S . A\right)$. In order to apply this model to skin both $A$ and $S$ must be identified. There are a number of ways of describing the origins of the interfacial shear stress for elastomeric materials, including a reversible peeling (adhesive) process involving the propagation of Schallamach waves. ${ }^{[31]}$ However, prediction of the interfacial shear stress is complex, requires detailed material property data and was beyond the scope of this project.

The deformation component $\mu_{\text {def }}$ arises from the incomplete recovery of the energy dissipated by subsurface viscoelastic deformation within the contact. ${ }^{[10]}$ Greenwood et al calculated that, for a sphere on a flat elastomeric surface, the friction coefficient due to deformation energy losses, $\mu_{d e f}$, is

$$
\mu_{d e f}=\alpha \frac{3}{16} \frac{a}{R}
$$

where $\alpha$ is the loss factor, $a$ the contact radius, and $R$ the ball radius. ${ }^{[32]}$ The loss factor, $\alpha=k \pi \tan \delta$, where $k$ is a constant and $\tan \delta$ the loss tangent, is the ratio of energy dissipated to the average energy stored per cycle in a deformation process and can be estimated from the loss tangent. For the PDMS/PDMS contact at the load used in this study, a contact radius of $1.65 \mathrm{~mm}$ was obtained using the JKR model. ${ }^{[29]}$ Assuming a value of $k=1$, which presumes a simple deformation cycle, ${ }^{[31]}$ a value of $\mu_{\text {def }}=0.01$ is obtained from Eq. 2 . The low value for $\mu_{\text {def }}$ compared to the value for the total friction coefficient of 0.44 , shows the overwhelming importance of interfacial adhesion in dry contact.

The low value for $\mu_{\text {def }}$ was also ascertained experimentally. As ball and disc are driven independently in the MTM, it is possible to run tests at any desired slide to roll ratio (SRR; defined as the ratio of sliding speed to entrainment speed) and to then separately determine the sliding friction coefficient and the rolling friction coefficient. ${ }^{[33]}$ To determine the rolling friction component for the tribological contact of interest, a test was carried out at the same load $(0.5 \mathrm{~N})$ and sliding speed $(50 \mathrm{~mm} / \mathrm{s})$ as described above, but instead of a SRR of 2.0 (pure sliding), an SRR of 1.8 was applied. A rolling friction coefficient of 0.03 was obtained, 
which is in the same order of magnitude as the predicted value and about two orders of magnitude less than the corresponding sliding friction coefficient, which was determined to be 0.48. Therefore, in this study, as in many other cases involving the friction of elastic and viscoelastic materials ${ }^{[34]}$ and friction on skin, ${ }^{[10]}$ the interfacial component dominates the overall friction coefficient.

For one experiment, presented in Fig. 8, the PDMS ball on the MTM was replaced with a Nylon one. A ca 6 times reduction in the overall friction coefficient was observed. This may be due to lower adhesion between Nylon and PDMS compared to PDMS on PDMS. But it will also be due to a difference in mechanical properties. Nylon has a higher Young's modulus (2.65 GPa) and lower Poisson's ratio (0.39). This combination will reduce the contact area to a calculated Hertzian contact radius of $1 \mathrm{~mm}$ and also the real area of contact. The stiffer contact and smaller area also reduce rolling friction, which is nearly halved to 0.0068 but, as for the PDMS/PDMS contact, the contribution to sliding friction from deformation is still small compared to the adhesive contribution.

\subsection{Friction by $\mathrm{SM}$ in fully flooded (FF) conditions}

When the contact is fully flooded with particle-free suspension medium (SM, FF), the friction coefficient is much lower than the un-lubricated case. The lubricant film thickness was not measured but was calculated using the Hamrock and Dowson ${ }^{[35]}$ equations for film thickness in elliptical contacts for materials of low elastic modulus. For the circular contact of interest in this study, these equations reduce to;

$$
\begin{aligned}
& h_{c}=3.3 \bar{U}^{0.64} \bar{W}^{-0.22} R^{\prime} \\
& h_{m}=2.8 \bar{U}^{0.65} \bar{W}^{-0.21} R^{\prime}
\end{aligned}
$$

where $h_{c}$ and $h_{m}$ are the central and minimum film thickness respectively and the dimensionless operating parameters are:

$$
\begin{aligned}
& \text { dimensionless speed parameter, } \bar{U}=\frac{U \eta}{E^{\prime} R^{\prime}} \\
& \text { dimensionless load parameter, } \bar{W}=\frac{W}{E^{\prime} R^{\prime 2}}
\end{aligned}
$$

where $U$ is the entrainment speed defined as the disc speed divided by $2, W$ the applied load, $\eta$ the lubricant dynamic viscosity, $R^{\prime}$ the reduced contact radius in the entrainment direction, 
and $E^{\prime}$ the reduced elastic modulus. The latter two terms are defined by $1 / R^{\prime}=1 / R_{x 1}+1 / R_{x 2}$ and $2 / E^{\prime}=\left(1-v_{1}^{2}\right) / E_{1}+\left(1-v_{2}^{2}\right) / E_{2}$, respectively, where $R_{x 1}, R_{x 2}, E_{1}, E_{2}, v_{1}$, and $v_{2}$ denote the radii in the entrainment direction, the Young's moduli, and the Poisson's ratios of the two contacting bodies. Using Equation (6) the minimum film thickness for the tribological contact of interest is calculated to be $1.35 \mu \mathrm{m}$. This is considerably greater than the out-of-contact roughness of the surfaces which suggests that in fully flooded conditions the contact will operate under full film lubrication with negligible solid-solid contact or interfacial adhesion. For a compliant contact this lubrication regime is known as the isoviscouselastohydrodynamic regime (I-EHL). Within this, regime the contact pressure is large enough to cause significant elastic deformation of one or both the contacting solids, but not great enough to cause any substantial change to lubricant viscosity. Equation (1) now become, for a fully flooded contact;

$$
\mu_{t o t}=\mu_{d e f}+\mu_{I-E H L}
$$

where $\mu_{I-E H L}$ is the friction coefficient formed by the hydrodynamic forces from lubricant flow. Using numerical solutions to the Reynolds and elastic deformation equations, de Vicente $e t a l^{[36]}$ developed regression equations to predict the separate contributions of flow on friction coefficient in a circular, ball-on-flat, I-EHL contact:

$$
\mu_{I-E H L}=\mu_{\text {Couette }}-\mu_{\text {Poiseuille }}
$$

where;

$$
\mu_{\text {Couette }}=\operatorname{SRR}\left(3.8 \bar{U}^{0.71} \bar{W}^{-0.76}+0.96 \bar{U}^{0.36} \bar{W}^{-0.11}\right)
$$

and,

$$
\mu_{\text {Poiseuille }}=1.46 \bar{U}^{0.65} \bar{W}^{-0.70}
$$

where SRR is the slide-roll ratio, defined as the ratio of the absolute value of the sliding speed, $u_{s}=\left|u_{b}-u_{d}\right|$, to the entrainment speed, $U=\left(u_{b}+u_{d}\right) / 2$, where $u_{b}$ and $u_{d}$ are the surface speeds of the ball and disc, respectively. $S R R$ can be given as:

$$
S R R=\frac{\left|u_{b}-u_{d}\right|}{0.5\left(u_{b}+u_{d}\right)}
$$


The Couette component of flow arises from the shear stresses created in the fluid film due to the surfaces moving relative to each other. Therefore, Couette forces have different sign for each surface depending upon the sign of the sliding speed. Whereas, the Poiseuille component results from shear stresses arising due to the fluid film pressure gradient, $\tau_{p}=$ $(h / 2) d p / d x$, where $h$ is the local film thickness and $d p / d x$ the pressure gradient in the entrainment direction. Poiseuille friction is present in both rolling and sliding contacts. It always has a net negative value, i.e. it hinders the surface moving relative to the contact. Hence the sign convention in Eq. (8).

A value of $\mu_{I-E H L}=0.0053$ was found for the test conditions $(S R R=2, W=0.5 \mathrm{~N})$. Using Equation (7) $\mu_{\text {tot }}$ is calculated to be 0.0138 which is reasonably close to the value of 0.012 presented in Fig. 3.

\subsection{Friction by $S M$ in partially lubricated (PL) conditions}

When the contact is only partially lubricated (PL) by SM, the initial value of the friction coefficient is similar to the friction coefficient value for the FF contact so a full I-EHL film is presumably present. This persists for about 200 seconds (stage 1) and is terminated by a sudden and rapid increase in friction. This is believed to result from the onset of starvation due to the loss of lubricant from the contact inlet reservoir. It was observed that the onset of stage 2 was highly sensitive to small changes in temperature and humidity, so evaporation is one mechanism for the loss of lubricant with time. The extremely rapid increase in friction suggests that dewetting of the surfaces may occur at the end of stage 1.

\subsection{Effect of suspended particles on friction under FF conditions}

Under FF conditions, the addition of particles had negligible influence on the value of the friction coefficient, regardless of their size or concentration. This is quite surprising since if particles pass through the contact, an increase in the hysteresis losses, and therefore the overall friction coefficient, should be observed. The possibility that the film thickness is larger than the suspended particle diameter can be discounted since the calculated film thickness, of ca $1.35 \mu \mathrm{m}$, is clearly smaller than the diameter of any of the tested particles, even allowing for some increase of viscosity of the fluid due to its particle content. Thus, it is likely that particles do not pass through the contact under FF conditions. The most likely explanation is that very few particles, if any particles actually enter and pass through the contact. On approaching the contact inlet, most suspended particles will be subjected to off-axis fluid 
forces as the majority of lubricant flows around the contact, not through it. Only particles very close to the central streamline will thus enter the contact. ${ }^{[37]}$ Dwyer-Joyce also suggests that there is a maximum size of particles that can be entrained into a contact due to geometry. ${ }^{[37]}$ This accords with the images in Fig. 9, where larger particles are observed to accumulate further from the contact region than smaller ones. Under PL conditions, any fluid forces will, of course, be lost as the SM evaporates, until only the surface forces between the particles and main contacting bodies act to entrain the particles into the contact.

\subsection{Effect of suspended particles on friction under PL conditions}

It is clear from Fig. 4 that, under PL conditions, the presence of solid particle influences each stage of the friction curve. The effect of particle size and concentration on the duration and magnitude of each stage is now discussed.

\subsubsection{Stage 1: initial low friction state}

An increase in particle concentration leads to a decrease in the duration of the initial low friction state, stage 1 (Fig. 6). An increase in particle size appears to have the same effect on stage 2 (Fig. 8). It is likely that the duration of these states is governed by the onset of starvation and the evaporation of the SM. Figure 5 shows that particles rapidly collect in, or near, the contact inlet. Figure 7 illustrates that an increase in concentration leads to a greater number of particles in the agglomeration. While it is difficult to see, Fig. 8 shows that an increase in size causes the agglomeration to occur further from the contact inlet. This is a result of contact geometry. These agglomerations of particles will have two effects; (i) to starve the contact of SM by blocking the flow of fluid into the contact, and (ii) to speed up evaporation of the SM by increasing surface area.

It can be seen in Fig. 6 that an increase in particle concentration leads to a decrease of the duration in the initial low friction state. Both concentration and particle size control lubricant SM phase volume fraction; defined as the percentage of test fluid that is SM. A decrease in SM phase volume fraction means a lower volume of SM present at the beginning of the test, as the same volume of suspension is employed for each test. When higher ratios of SM are present, evaporation should take longer, and the increase in friction should occur later.

To test this hypothesis, the duration of the initial low friction state was plotted versus the amount of SM in the suspensions that were applied on the disc in each case. For comparison, 
results for the particle-free SM are also presented where different amounts of the SM were applied to the disc for each test. Figure 10 presents the results.

In the absence of particles, the duration of the initial low friction state increases linearly with volume of SM applied to the disc. However, for suspensions, a highly non-linear rise in stage 1 with SM volume was observed. This indicates that the particles actively promote the termination of the full I-EHL stage 1. This may be because they block the inlet, preventing fluid entrainment.

\subsubsection{Stage 2: increasing friction coefficient}

Figure 4 shows that initial friction coefficient values are similar to fully-flooded measurements. It is likely that the contact is operating within the I-EHL regime during this period. Once starvation has started, the film thickness will decrease until the surfaces are close enough for asperity interaction to occur and friction will then rise. The length or duration of this stage (2) is now determined by the rate of complete removal of the SM from the contact track, most likely via evaporation.

It was found that the duration of stage (2) increased with decreasing particle size at a fixed overall powder concentration. At a given powder concentration, as particle size is decreased the number of particles present in a given volume of suspension will increase. It is probable that SM is held between particles by surface tension and this slows evaporation of the SM. This is in accord with the powder-free results which show the shortest stage (2) duration.

Figure 6 shows the effect of particle concentration on the rate of the friction coefficient transition. The power law gradient of each slope is presented in Table 1; the slope is defined to start at the initial friction coefficient increase until a upper value of $\mu=c a 0.3$ is reached, where all concentrations are observed to plateau. As particle concentration, and therefore particle number, is increased the rate of transition slows.

\subsubsection{Stage 3: friction coefficient plateau}

Once operating within stage 3 , where friction has levelled out, it is believed that the contact is void of SM so that any differences in friction behaviour originate only from solid particles. Figures 4, 6 and 8 show that the presence of particles within the contact reduces the friction coefficient, compared to the un-lubricated contact. The high friction coefficient observed for the un-lubricated contact has been ascribed to strong interfacial adhesion between the PDMS 
surfaces. Figure 8 shows that for a pure PDMS/Nylon contact, friction is greatly reduced compared to a pure PDMS/PDMS contact. Equation (2) indicates that the friction coefficient due to deformation losses will drop due to the higher Young's modulus of Nylon. However, the deformation losses for the dry PDMS/PDMS contact are small and cannot account for the large drop in friction coefficient. Therefore it is safe to assume that the reduction is due to a change in the interfacial adhesion. Interfacial adhesion is proportional to the real contact area and the interfacial shear stress and the introduction of the Nylon particles may reduce both.

To examine the effect on contact area of particles in a contact, an optical technique was used to capture images from a PDMS/glass contact. Hemispherical PDMS samples were moulded using a concave lens (Edmund Optics Inc., USA) with a radius of curvature of $12.9 \mathrm{~mm}$. Since PDMS is transparent, reflections from within the specimen or the metallic sample mounting will interfere with the signal of interest so a small proportion of opaque filler was added to the PDMS to prevent this. Carbon black (Fluffy; Cabot Corporation, USA) at a concentration of $0.5 \%$ wt. was added to the PDMS solution prior to curing.

Using a deadweight lever arm, the PDMS samples were loaded against a flat glass disc, and images captured of the contact area between the two surfaces. Figures 11 A) and B) show the contact under zero load and $0.5 \mathrm{~N}$, in the absence of Nylon particles. The loaded contact region, (Fig. $11 \mathrm{~B}$ ), appears as a dark, circular patch. The contact area between the PDMS hemisphere and the glass disc was also measured in the presence of Nylon particles. To do this, a small volume of powder was placed on the PDMS and then air-blown to remove particles that did not adhere to the surface.

Figure $11 \mathrm{C}$ ) shows the particles visible on PDMS surface at zero load. When the load is increased to $0.5 \mathrm{~N}$, Fig. $11 \mathrm{D})$, a quite different contact is visible compared to Fig. $11 \mathrm{~B}$ ). Deformed areas appear grey. These grey areas are believed to represent regions where the PDMS separates from the glass as the relatively rigid particles support the load and push the PDMS substrate away from the glass. The real area of contact between glass and Nylon particles is too small to detect. When the load is increased further, to $1 \mathrm{~N}$, clear black regions begin to appear, as shown in Fig. 11 E). These correspond to areas of direct PDMS/glass contact. At a load of $10 \mathrm{~N}$, the majority of the PDMS surface is in contact with the glass, as seen in Fig. $11 \mathrm{~F})$. The lighter areas represent regions where PDMS and glass are separated because the PDMS is being pushed away from the glass by the load-bearing solid particles. 
Even though the images show a PDMS/glass contact, it is likely that the behaviour will be broadly similar between two PDMS surfaces.

Figure 11 shows that the presence of solid particles in the contact reduces the real contact area by supporting some of the load and pushing regions of the PDMS away from the glass. Figure 6 shows that the number of particles in the lubricant has minor effect on the number of particles able to enter the contact during stage 3. The major factor in reducing boundary friction through the presence of solid particles is particle size, as shown clearly in Fig. 8. This is due to contact geometry, not due to lubricant flow.

\section{Summary and conclusions}

A ball-on-disc tribometer has been used to examine a compliant PDMS/PDMS contact lubricated with Nylon particles suspended in an aqueous medium. The aim of this investigation was to mimic the application of cosmetic products on human skin. The effects on friction of varying particle concentration and size were investigated. Tests were conducted at room temperature for 20 minutes at a constant sliding speed of $50 \mathrm{~mm} / \mathrm{s}$.

A friction coefficient of $c a 0.44$ was observed for un-lubricated PDMS/PDMS. The addition of lubricant, with or without particles, lowered the friction coefficient. Under fully-flooded conditions no significant differences were detected between lubricants regardless of particle presence, size or concentration. It is believed that under these conditions a full I-EHL liquid film is present in the contact and most particles, if present, fail to enter the contact. For partially lubricated contacts, where only a very small volume of lubricant is supplied at the start of the test, a three stage friction coefficient curve was observed for all experiments;

- Stage 1; an initial low friction state, corresponding to that seen in fully-flooded conditions

- Stage 2; a transition period where friction rises rapidly with rubbing time

- Stage 3; where friction coefficient levels out at a constant, relatively high value.

As particle concentration was increased the duration of stage 1 decreased until, for concentrations of $\geq 25 \% \mathrm{wt}$, friction coefficient began to rise as soon as the test was started. It is believed that the transition from stage 1 to stage 2 results from lubricant starvation, which 
occurs due to evaporation of the aqueous medium and, when particles are present, by the accumulation of particles in the contact inlet.

The duration of stage 2 was observed to increase with increasing particle concentration and decreasing particle size. This was tentatively related to the number of particles present on the surfaces, which may promote evaporation of the aqueous medium.

Stage 3 occurred after full evaporation of the aqueous suspension medium. Images of the contact region taken post test showed that, at this stage, the majority of particles accumulate around the contact region. Only a small number of particles are present in the tribological contact. It is believed that these particles lead to a reduction in dry friction compared to the un-lubricated contact between two PDMS surfaces. Optical images showing the real area of contact between a PDMS and a glass surface support this mechanism by showing that solid particles of Nylon, which is stiffer than PDMS, reduce the contact area between the two surfaces. Interfacial adhesion and, thus, friction, are reduced. The initial particle concentration was observed to have little effect on the value of the final friction coefficient. Probably because most particles failed to enter the contact. A significant increase in the friction coefficient in stage 3 was observed with an increase in particle size, because smaller particles are more likely to enter the contact.

These findings show that the properties of solid particles in aqueous suspensions can significantly influence friction in compliant contacts and that frictional behaviour is highly dependent on the behaviour of the particles and the way that they become distributed on the surfaces. This is believed to have implications in the design of effective, powder-containing cosmetics.

\section{Acknowledgements}

The authors wish to thank Dr Nils Hoffmann and Dr Margaret Horton (Beiersdorf AG) for inspiring discussions; Stefan Wiesner (Beiersdorf AG) for support with the SEM images; and Dr Karine Loyen and Hélène Egret (Arkema Group) for supplying the Nylon particles. The authors are grateful to Beiersdorf Research for permission to publish these results.

\section{References}

[1] Underwood, R. The tribological effects of contamination in rolling element bearings. London: London University Press; 2008. 
[2] Holinski, R. The influence of boundary layers on friction. Wear. 1979; 56(1): 147-154.

[3] Brummer, R. Godersky, S. Rheological studies to objectify sensations occuring when cosmetic emulsions are applied to the skin. Colloids and Surfaces A: Physicochemical and Engineering Aspects. 1999; 152(1-2): 89-94.

[4] Gee, M.G. et al. A new friction measurement system for the frictional component of touch. Wear. 2005; 259 (7-12): 1437-1442.

[5] Lodén, M. et al. Instrumental and sensory evaluation of the frictional response of the skin following a single application of five moisturizing creams. Journal of the Society of Cosmetic Chemists. 1992; 43(1): 13-20.

[6] Gitis, N. Sivamani, R. Tribometrology of skin. Tribology Transactions. 2004; 47: 1-9.

[7] Prall, J. K. Instrumental evaluation of the effects of cosmetic products on skin surfaces with particular reference to smoothness. Journal of the Society of Cosmetic Chemists 1973; 24: 693-707.

[8] Cussler, E. L. Zlotnick S. J. Shaw, M. C. Texture perceived with the fingers. Perception and Psychophysics 1977; 21(6): 504-512.

[9] Nacht, S. et al. Skin friction coefficient: changes induced by skin hydration and emollient application and correlation with perceived skin feel. Journal of the Society of Cosmetic Chemists. 1981; 32: 55-65.

[10] Adams, M. Briscoe, B. Johnson, S. Friction and lubrication of human skin. Tribology Letters. 2007; 26(3): 239-253.

[11] Highley, D. R. et al. Frictional properties of skin. The Journal of Investigative Dermatology. 1977; 69: 303-305.

[12] Naylor, P. F. D. Experimental friction blisters. The British Journal of Dermatology. 1955; 67(10): 327-342.

[13] Comaish, S. Bottoms, E. The skin and friction: Deviations from Amonton's laws, and the effects of hydration and lubrication. The British Journal of Dermatology. 1971; 84: 37-43.

[14] Appeldoorn J. K. Barnett, G. Frictional aspects of emollience. Proceedings of the Scientific Section of the Toilet Goods Association. 1963; 40: 28-35.

[15] El-Shimi, A. F. In vivo skin friction measurements. Journal of the Society of Cosmetic Chemists. 1977; 28: 37-51.

[16] Gerrard W. A. Friction and other measurements of the skin surface. Bioengineering of the Skin. 1987; 3(2): 123-139.

[17] Myant, C. Experimental techniques for investigating lubricated, compliant, contacts. London: Imperial College London; 2010.

[18] Scheibert, J. Leurent, S. Prevos, A. Debregeas, G. The role of fingerprints in the coding of tactile information probed with a biomimetic sensor. Science. 2009; 323(5920): 1503-1506.

[19] Bongaerts, J. H. H. Fourtouni, K. Stokes, J. R. Soft-tribology: Lubrication in a compliant PDMS-PDMS contact. Tribology International. 2007; 40(10-12): 15311542 .

[20] Agache, P. Humbert, P. Measuring the skin. Berlin: Springer-Verlag; 2004.

[21] Myant, C. Spikes, H. A. Stokes, J. R. Influence of load and elastic properties on the rolling and sliding friction of lubricated compliant contacts. Tribology International. 2010; 43(1-2): 55-63.

[22] Myant, C. Reddyhoff, T. Spikes, H. A. Laser-induced fluorescence for film thickness mapping in pure sliding lubricated, compliant, contacts. Tribology International. 2010; 43(11): 1960-1969.

[23] Callister, W. D. Materials Science and Engineering. An Introduction. New York: John Wiley and Sons; 2000. 
[24] Al-Malah, K. Rheological properties of carbomer dispersions. Annual Transactions of the nordic Rheology Society. 2006; 14: 123.

[25] Zhu, H. Kim, Y. D. De Kee, D. Non-Newtonian fluids with a yield stress. Journal of Non-Newtonian Fluid Mechanics. 2005; 129(3): 177-181.

[26] Tadros, T. F. Rheology of Dispersions. Weinheim: Wiley-VCH; 2010.

[27] Chow, T. S. Shear thinning in concentrated dispersions. Journal of Physics: Condensed Matter. 1996; 8(43): 8145.

[28] Johnson, K. L. Kauzlarich, J. J. Transfer of material between rolling and sliding surfaces. International Journal of Mechanical Sciences. 2004; 46(3): 343-357.

[29] Johnson, K. L. Kendall, K. Roberts, A. D. Surface energy and the contact of elastic solids. Proceedings of the Royal Society of London. A. Mathematical and Physical Sciences. 1971; 324(1558): 301-313.

[30] Bowden, F. P. Tabor, D. The friction and lubrication of solids. Oxford: Oxford University Press; 1954.

[31] Johnson, S. A. Gorman, D. M. Adams, M. J. Briscoe, B. J. The friction and lubrication of human stratum corneum. Tribology Series. 1993; 25: 663-672.

[32] Greenwood, J. A. Minshall, H. Tabor, D. Hysteresis losses in rolling and sliding friction. Proceedings of the Royal Society of London. Series A. Mathematical and Physical Sciences. 1961; 259(1299): 480-507.

[33] de Vicente, J. Stokes, J. R. Spikes, H. A. Lubrication properties of non-absorbing polymer solutions in soft elastohydrodynamic (EHD) contacts. Tribology International. 2005; 38(5): 515-526.

[34] Wolfram, L. J. Friction of skin. Journal of the Society of Cosmetic Chemists. 1983; 34: 465-476.

[35] Hamrock, B. J. Dowson, D. Isothermal elastohydrodynamic lubrication of point contacts. Part III - fully flooded results. Trans. ASME, Journal of Lubrication Technology. 1977; 99(2): 264-276.

[36] de Vicente, J. Stokes, J. R. Spikes, H. A. The frictional properties of Newtonian fluids in rolling-sliding soft-EHL contacts. Tribology Letters. 2005; 20(3-4): 273-286.

[37] Dwyer-Joyce, R. S. The effects of lubricant contamination in rolling element performance. London: University of London Press; 1993 


\section{Figure captions}

Figure 1: Schematic diagram of the MTM

Figure 2: SEM images of (a) Nylon-12 particles of $21.8 \mu \mathrm{m}$ and (b) Nylon-12 particles of 63.2 $\mu \mathrm{m}$

Figure 3: Friction coefficient versus time is shown for the PDMS/PDMS tribological contact under dry conditions (black circles) and lubricated by the SM under FF (blue squares) and PL (red triangles) conditions.

Figure 4: Friction coefficient versus time is shown for PDMS/PDMS lubricated with powder suspension under FF and PL conditions. Letters (a) to (e) indicate stages were the test was stopped and photomicrographs of the contact taken. Particle-free plots from Fig. 3 are shown as solid or dashed lines.

Figure 5: Images of the ball and disc in the tribological contact of interest, lubricated with the powder suspension. Contact inlet is on the left in all images. Letters (a) to (e) indicate the corresponding stages of the curve shown in Fig. 4.

Figure 6: Friction coefficient versus time is shown for the tribological contact of interest under PL conditions. A suspension of Nylon particles, $21.8 \mu \mathrm{m}$ diameter at various concentrations was used for all test lubricants. Particle-free results from Fig. 3 are shown as solid or dashed lines.

Figure 7: Images of the ball post test, at $\mathrm{t}=1200 \mathrm{~s}$. The contact was partially lubricated with suspensions of Nylon particles $(21.8 \mu \mathrm{m}$ diameter) at 10, 20, and $30 \%$ wt concentration. Contact inlet is on the left in all images.

Figure 8: Friction coefficient versus time for the tribological contact of interest partially lubricated with $30 \%$ wt concentration powder suspensions. Three particle diameters are presented; 11.5 (red circles), 21.8 (green triangles) and 63.2 (blue squares) $\mu \mathrm{m}$ diameter. Also shown is the result for the Nylon ball on PDMS disc (yellow diamonds). Master plots from Fig. 3 are shown as solid or dashed lines.

Figure 9: Images of the ball (top) and disc (bottom) contact region taken post test, at $\mathrm{t}=1200$ s. Initially the contact was lubricated under PL conditions with suspensions of Nylon particles of 11.5, 21.8 and $63.2 \mu \mathrm{m}$ diameter at $30 \%$ wt concentration. 
Figure 10: Duration of stage 1 versus amount of SM applied to the disc (100\% correspond to $25 \mu 1)$.

Figure 11: Images of the real area of contact in the presence and in the absence of particles (Nylon, $21.8 \mu \mathrm{m}$ diameter) at various loads 


\section{Tables:}

Table 1: Power law gradient versus powder concentration for all suspension of Nylon particles, $21.8 \mu \mathrm{m}$ diameter, presented in Fig. 6 .

\begin{tabular}{l|cccccc}
\hline Concentration $(\% \mathrm{wt})$ & 0 & 5 & 10 & 20 & 25 & 30 \\
\hline Power law gradient & 7.71 & 5.01 & 4.61 & 1.64 & 1.26 & 0.8 \\
\hline
\end{tabular}

